\title{
Mesh Simplification Using the Edge Attributes
}

\author{
II Dong Yun \\ School of Electronics and Information Engineering, Hankuk University of F. S., Yongin 449-791, Korea \\ Email:yun@hufs.ac.kr
}

Kyowoong Choo

Motorola Korea Design Center, Seoul 135-766, Korea

Email:w19664@motorola.com

\author{
Sang Uk Lee \\ School of Electrical Engineering, Seoul National University, Seoul 151-742, Korea \\ Email:sanguk@sting.snu.ac.kr
}

Received 14 November 2001 and in revised form 15 February 2002

\begin{abstract}
This paper presents a novel technique for simplifying a triangulated surface at different levels of resolution. While most existing algorithms, based on iterative vertex decimation, employ the distance for error metric, the proposed algorithm utilizes an edge criterion for removing a vertex. An interior angle of a vertex is defined as the maximum value of all possible angles formed by combinations of edges connected to a vertex. Since the surface curvature examined with the interior angle provides more information for decision of vertex removal than the conventional distance measure, the proposed algorithm can approximate the surface with less computation. The height of a triangle, which is formed by the pair of edges, is also used for an additional constraint. The computational complexity can thus be greatly alleviated to logarithmic scale from the exponential scale required for the conventional algorithms, while yielding the comparable error level.
\end{abstract}

Keywords and phrases: LOD, level of detail, mesh simplification, multiresolution.

\section{INTRODUCTION}

Detailed mesh data models, obtained from range scanning systems, are too dense for practical applications. Transmission and storage requirements of such $3 \mathrm{D}$ graphic models are very demanding, due to the large amounts of data [1]. While rendering performance is continually improving, further improvement in performance could be possible by adapting the complexity of a model to its contribution to the rendered image. The ideal solution will efficiently determine the coarsest model, while retaining the perceptual image qualities.

One common heuristic technique is to author several versions of a model at various level of detail (LOD); a detailed triangle mesh is used when the object is close to the viewer, and coarser approximations are substituted as the viewing distance increases. The LOD assigns multiple models varying in resolution for an object. Thus, virtual reality (VR) machines can render objects in a virtual world according to their proper resolution, without displaying all the polygons in full detail.

The multiresolution modeling, motivated by such requirements, approximates high-density models nearly indistinguishably with fewer faces for rendering efficiency [2].
This is accomplished by iteratively removing vertices from original faces that will not significantly degrade the global shape of the model. The distance from the original surface to the reformed one usually represents the error caused by removing each vertex. Most existing algorithms mainly differ in their order of choosing a vertex for removal. However, heavy computation is required for estimating the consequential errors caused by removing a vertex. These approaches cannot provide much information about the surface, while they can be implemented easily. Edge contraction as well as vertex decimation, to be introduced in Section 2, falls into this category. Edge contraction collapsing vertices, lying on both sides of an edge, lacks diversity in the simplification of a given region, because pulling both ends of an edge to the middle is the only required operation.

In this paper, we focus on introducing a simple primitive for vertex decimation that could be implemented without heavy computational burden. By evaluating the surface curvature with edge or face information, better results could be obtained, since the local curvature is the most important information for decimation. An algorithm utilizing the angle between the faces has already been reported [3]. When 
the surface curvature of adjacent triangles are evaluated and sorted to collapse the centering triangle's three vertices to a single point, all regions connected are affected, resulting in significant variations in surface structure.

The edge, on the other hand, contains enough-if not complete-information about the local surface surrounding a vertex, while keeping the variation small. The surface information can be easily obtained by evaluating the interior angles of the surrounding edges. Hence, by adopting an edge as a primitive, a vertex to be removed can be selected quickly, reflecting the surface information as well. While the existing algorithms consume much time in whether or not to remove a vertex by actually removing and evaluating the resulting error, the proposed approach works deterministically by removing vertices in the order of their height and angle without further analysis. In addition, by filling the hole with Delaunay triangulation, we can give more degree of freedom than the conventional edge collapse approaches in the retriangulation of simplified area. Therefore, both the computational load and surface estimation errors can be reduced by utilizing the edge information.

\section{PREVIOUS APPROACHES IN MESH SIMPLIFICATION}

The approaches to be introduced are minimum number vertex-based approximations. For this kind of the approximation problem, we consider the given error bound $\epsilon$. Then the objective is to minimize the number of vertices such that no point of the approximation is further away than $\epsilon$ from the input model [4]. As will be discussed in Section 2.1, most approaches rely solely on the vertex error with perhaps one or more additional constraints. This can only give obscure information about the surface to be approximated, yielding to a simplified object whose errors are minimum in numeric domain only, which is not visually optimum.

\subsection{Vertex decimation}

Vertex decimation is an iterative surface simplification approach $[5,6,7]$. In each step, when a vertex is selected for removal, all faces adjacent to that vertex are removed from the model, then the resulting hole is retriangulated. Because the main purpose of these algorithms is to reduce the density of acquired meshes, we should be careful to preserve the topology.

One of the first iterative mesh simplification algorithms was proposed by Lorensen et al. [5], in which the vertices are removed from the mesh, and the local neighborhood surrounding the point is retriangulated on the local plane of the vertex. A point is removed when the distance to the best-fit plane of the surrounding point is small. All vertices with an error that is less than a threshold and satisfying topology preserving condition, are removed. Since primitives are not ordered for decimation, a vertex with greater error may be removed first.

Soucy and Laurendeau $[6,7]$ presented a sequential optimization algorithm, in order to remove the vertex that minimizes the retriangulation error after each iteration. The

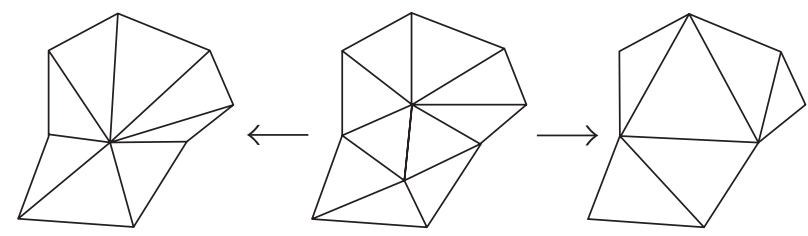

Edge collapse

Vertex decimation

Figure 1: Comparison of freedom of retriangulation between edge collapse and vertex decimation.

purpose of the sequential algorithm is to remove the maximum number of vertices, while keeping the triangulation error as low as possible. The equi-angularity of surface triangulation is optimized in 3D space throughout the sequential process, by using an unconstrained Delaunay triangulation algorithm [8]. The main disadvantage of these algorithms, however, is that the cost of computation is very expensive. Since it needs to evaluate all errors from previously removed vertices, the computational complexity increases as the process goes on. In general, the computation is proportional to the exponential scale.

\subsection{Edge contraction}

An edge contraction takes two endpoints of a target edge, moves them to the same position, links all the incident edges to one of the vertices, deletes the other vertex, and removes any faces that have degenerated into lines or points. These algorithms iteratively contract the edges of the model. The primary difference lies in how to choose the particular edge to be collapsed $[9,10,11]$.

Guéziec's [9] mesh simplification algorithm improves Schroeder's algorithm in many ways. Guéziec employs edges as the mesh primitive and edge collapse to eliminate retriangulation from the mesh simplification algorithm. The edges are ordered based on edge length, and a single pass through the edges is performed. During edge collapse, a new vertex is created. As in most approaches, Guéziec's simplification method bounds the total change in mesh shape. However, mesh shape is bounded using a complex construction called a tolerance volume, whose update requires a dynamic programming approach. Furthermore, Guéziec's algorithm cannot explicitly control the resolution of the generated meshes and cannot handle the vertices along the boundary of a mesh to prevent shrinking during simplification.

Hoppe et al. [10] iteratively optimizes an energy function over a mesh to minimize both the distance of the approximating mesh from the original, as well as the number of approximating vertices. And it is based on edge collapse, edge swap, and edge split to iteratively refine an initial mesh that is close to a set of 3D data points. Mesh optimization can handle free form as well as polyhedral objects. Mesh optimization requires three nested loops and is subsequently quite slow in computation, but can produce very concise and accurate meshes. However, the edge collapse lacks versatility in the selection of collapsing point by limiting it to lie in the center of two end points to be collapsed. Figure 1 compares 
the degree of freedom for retriangulation between edge contraction and vertex decimation approaches.

\subsection{Remarks}

This section presents several vertex-based multiresolution modeling techniques, each of which has its own input criteria and advantages or disadvantages. The vertex-based algorithm is mainly based on vertex decimation and edge contraction approaches. Both evaluate the resulting error (or energy functions) caused by removing each vertex, then decide whether or not to allow the decimation (contraction). This error estimation should be repeated after each iteration, making it computationally expensive.

In addition, a face-based approach, utilizing the surface curvature between adjacent triangles, is not adequate for preserving topology, since it affects unnecessary larger area, in order to preserve the triangular structure. In this paper, an attempt is made to utilize both the curvature and the distance (error) information, while preserving the topology and yielding small numerical errors.

\section{EDGE-BASED MESH SIMPLIFICATION}

Our main aim is confined to vertex decimation-based multiresolution modeling, on the notion that vertex decimation guarantees zero error on remaining vertices, because it does not move vertices' position. Among the well-known vertex decimation-based algorithms, Soucy's vertex removal algorithm could be one of the best approaches, since the resulting error by removing a vertex is re-calculated and sorted after each iteration. Although Soucy's method seems to be reasonable at each step, it also cannot provide the global optimal solution for removing a given number of vertices for minimum error. In addition, the computational complexity is very expensive, since the error evaluation is required for every iteration.

Soucy's approach can be classified as the distance-based vertex removal. However, the distance itself may not be a good primitive for curvature estimation in surface simplification. The proposed approach is based on simple notion that high curvature regions are preserved and low curvature regions simplified. Therefore, by using both curvature and distance as primitives, better results could be expected.

\subsection{Edge-based vertex removal algorithm}

The proposed edge-based vertex removal algorithm utilizes curvature and distance information for evaluating the sequence of reduction. An angle between adjacent edges provides an approximate curvature of the surface lying on that edge pair. An interior angle of a vertex is defined as the maximum value of all possible angles between edges connected to a vertex.

In Figure 2a, a vertex in the center is connected by six neighboring vertices to form a hexagon when seen from above. If the interior angle between a pair of edges is close to $180^{\circ}$, it can be assumed that this pair may not distort the global shape significantly, when substituted by a single

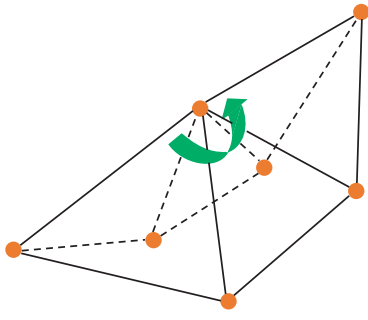

(a) Original edge pair.

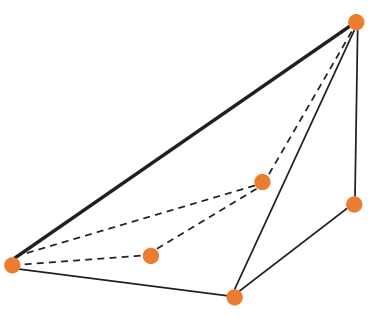

(b) Merged edge pair.
FIGURE 2: Removal of a vertex lying on a semi-straight edge pair.

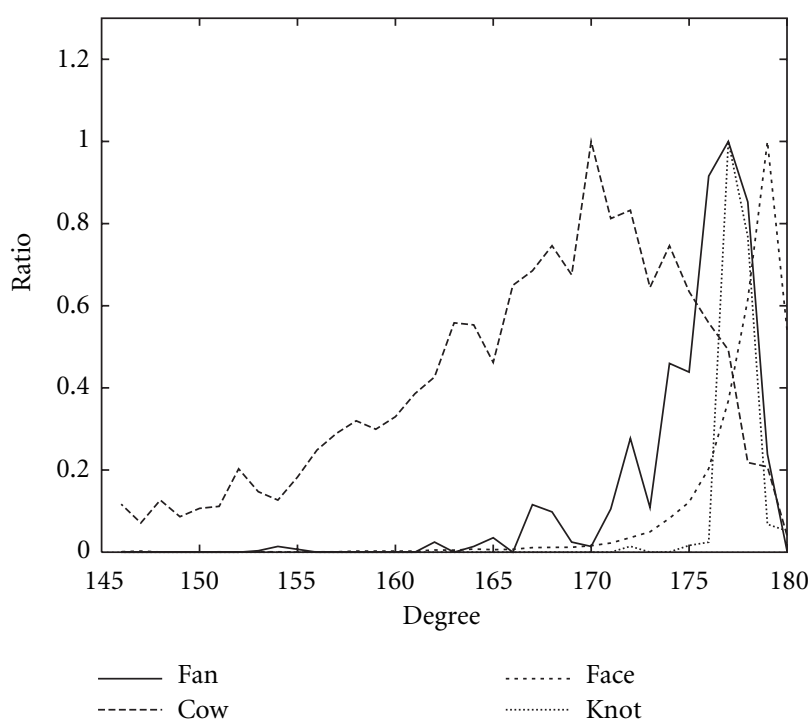

Figure 3: Histogram of interior angles.

edge. Hence, the vertex in the center qualifies to be in the queue of removal. Figure $2 b$ shows the vertex removing and re-meshing by Delaunay triangulation. In this case, a pair of edges whose angle is close to $180^{\circ}$ is replaced by a single edge drawn in thick line, then re-meshing procedure is performed on both sides of the edge. In order to justify the assumption, in our approach, the statistics of the interior angle distribution of the following 3D data are considered.

(i) The Fan, Face, and Knot data which are acquired from the STL objects library at ftp://fantasia.eng.clemenson.edu/ SLA/STL_objects.

(ii) The Cow data which was acquired from http:// almond.srv.cs.cmu.edu/afs/cs/user/garland.

Figure 3 shows the ratio of vertex number, revealing that more than $98 \%, 87 \%, 99 \%$, and $100 \%$ of vertices form angle pairs that yield larger angles than $160^{\circ}$ for Fan, Cow, Face, and Knot, respectively. This observation justifies the assumption of vertex removal based on edge angles, because great portions of edge pairs are indeed almost a straight line. Hence, the angle threshold which can be controlled by the user, is an important parameter in the proposed algorithm. 


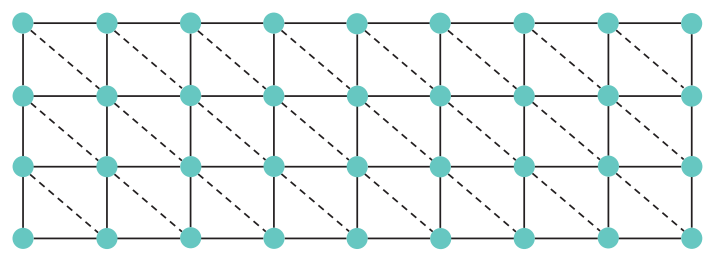

FIgURE 4: Initial triangulation of a dense range data.

However, the proposed algorithm includes a retriangulation process that may not preserve the surface contour of original model when applied inappropriately. Fortunately, the merged edge prevents this variation by constraining the area of retriangulation. While the previous algorithms retriangulates the whole area of vertex decimation, the proposed algorithm divides the area by two with the merged edge lying across it, constraining the region of retriangulation into smaller parts. Detailed description is presented in the following subsection.

There would be many retriangulation solutions, depending on the number of edges linked with the vertex. In advance, it is necessary to examine the distribution of edge numbers connected to a vertex. Normally, a dense range data, acquired from laser scanners, usually form a regular grid structure. The surface triangles can be easily made, by simply connecting vertices in horizontal and vertical directions to form a quadrangle, and by adding a diagonal line to divide this quadrangle into a triangle. An example is illustrated in Figure 4. Thus, it is easy to show that a vertex on a regular grid have six adjacent vertices connected by an edge. Experimentally obtained distribution of a connected number of edges agrees to our expectation. The number of connection exhibits a Gaussian-like distribution with mean $\approx 6$ as shown in Figure 5.

\subsection{Edge-constrained retriangulation process}

In large, the retriangulation process consists of two steps, depending on the adjacency of edge pair. The first is when the pair is not adjacent, and the other is when the edges are right next to each other. We begin with the adjacent pair, providing Figure 6 for visual demonstration of the following descriptions. The adjacent pair drawn in thick lines in Figures $6 \mathrm{~b}, 6 \mathrm{~d}$, and $6 \mathrm{e}$ shall be merged into an outer boundary edge, which is referred to as a boundary condition, denoted by "b." The boundary condition occurs quite often, due to the recursive nature of the proposed approach. The problem of how the holes in Figures 6b, 6d, and 6e should be re-meshed by triangles is intensively studied by [7]. The main approach is filling the hole with Delaunay triangles which contains the most equi-angular set of triangles [12]. This criterion avoids long narrow triangles that are numerically unstable. A simple algorithm introduced by Sibson [8] iteratively searches for adjacent triangles and swaps the diagonal (common edge) eventually converging to a Delaunay triangulation of the data points. Here, we use the interior angles of the triangles in $3 \mathrm{D}$

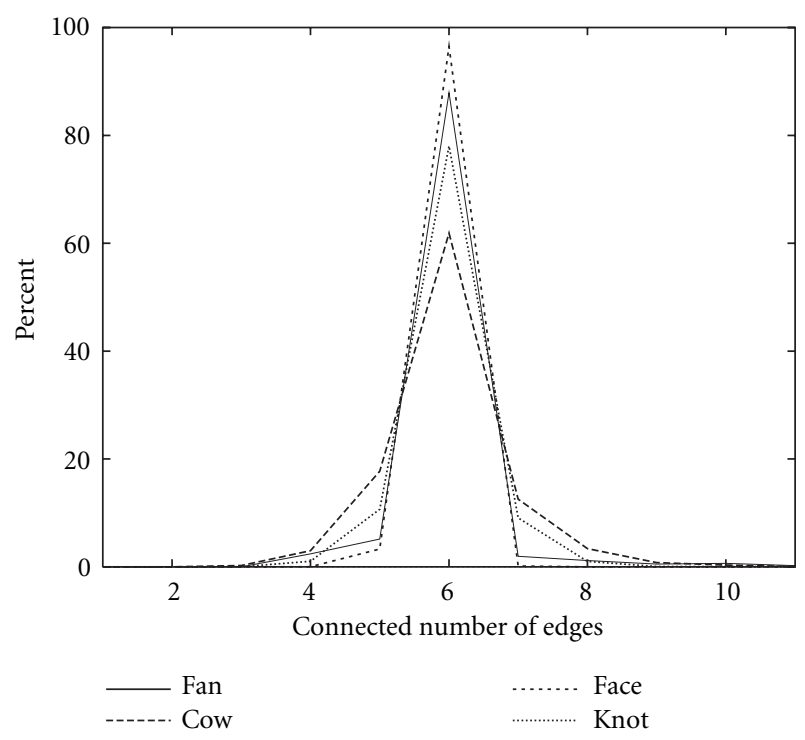

FIgURE 5: Histogram of edges linked to a vertex.

space as a metric for equi-angularity test, which is referred to as $2 \frac{1}{2} \mathrm{D}$ triangulation [6].

The nonboundary conditions are the dominating events in the retriangulation process. Figure 6f of "Case 6" is a good example of the nonboundary condition. When the centroid is removed and the thick edge pair is substituted by a single edge, two areas remain to be retriangulated, which is sent recursively back to "Case $4 \mathrm{~b}$," respectively. Then, "Case $4 \mathrm{~b}$ " retriangulates each region with iterative edge swapping operation of Sibson's. In Figure 6a, "Case 3" is actually a special case where the elimination of the centroid does not depend upon the angle, because there is no angle to compare with. Since we have focused the proposed algorithm with an additional distance criterion described in Section 3.3, no further manipulation of data is necessary, except for the deletion of the centroid from data list.

\subsection{Enhancement with additional constraint}

The approach discussed in Section 3.2 might not provide the satisfying results, because the proposed approach solely focuses on fast algorithm for vertex removal. However, it is worthy to note that there are long edges still abundant in the reduced model. Thus, a criterion to reduce the long edges should be considered to enhance the overall performance. We consider a height parameter $\delta$ for the angle criterion when the interior angles are pre-calculated. As depicted in Figure 7, the height parameter is the height of a triangle made of two points at the end of each edge and the vertex in the center. As mentioned previously, "Case 3" is merged if the distance from the vertex to the surrounding triangle is less than the threshold $\delta$.

The proposed algorithm works unsupervised with the maximum threshold height $\delta$ as the criterion for termination. It works as a double nested loop where the outer loop 


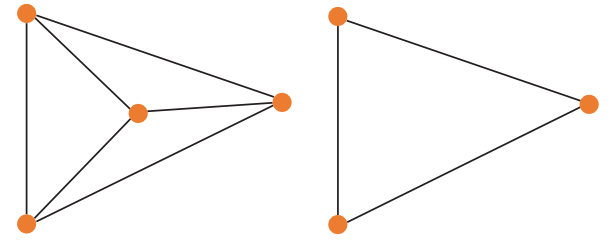

(a) Case 3 .
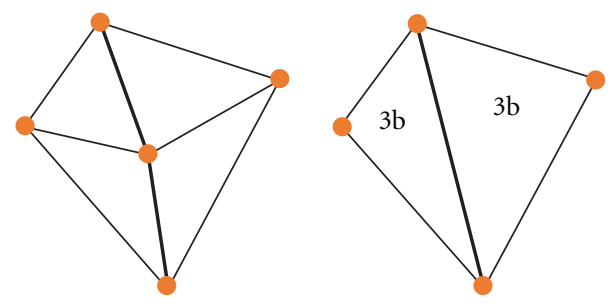

(c) Case 4 .
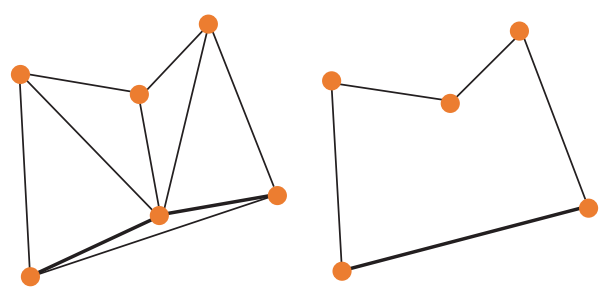

(e) Case 5b.

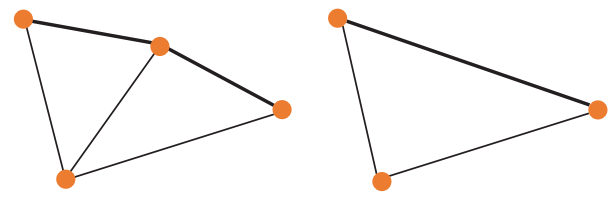

(b) Case 3b.

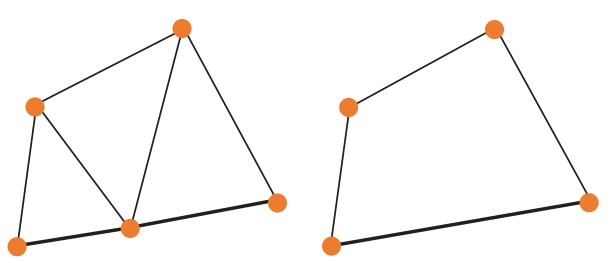

(d) Case 4b.
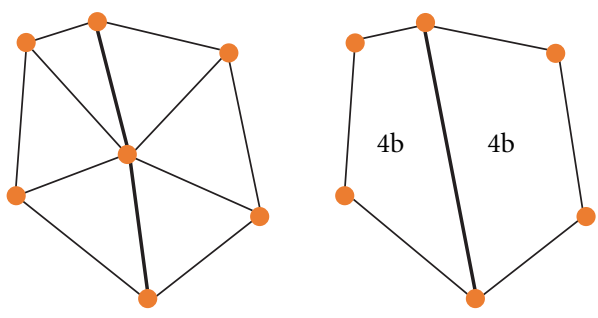

(f) Case 6.

FIgURE 6: Classification of the retriangulation process.

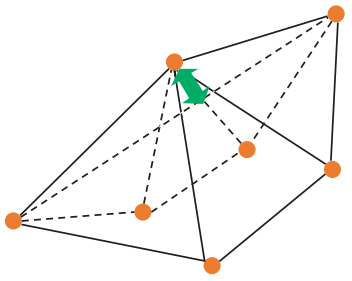

(a)

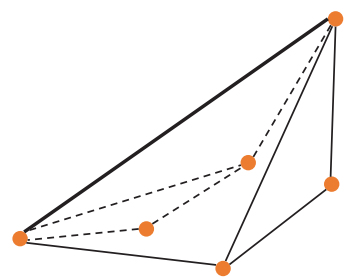

(b)
Figure 7: Additional height constraint $\delta$.

increases the height $\lambda$ from 0 to $\delta$, while the inner loop decreases the angle $\theta$ from $180^{\circ}$ to $\theta_{\min }$. Thus, an edge pair with larger interior angle, has a priority when two or more edge pairs exist having the same height. When the minimum value $\left(\theta_{\min }\right)$ of the inner loop is set to zero, small eruptions on the surface may be deleted. This is absolutely normal in global curvature sense, since small eruptions on a surface will not be seen from a distance. However, to preserve these features, we only need to increase $\theta_{\min }$ in the inner loop from zero to higher value. The experiments show empirically that $\theta_{\min }=150^{\circ}$ is sufficient for this purpose.
Swapping the outer and inner loops in the proposed algorithm may seem interesting. This can be reduced to the fundamental question of whether an edge pair with large interior angle has priority over the other edge pair which has the same height $\lambda$, but has smaller interior angle. It is concluded that the problem should be examined over the area affected by removal. Removal of pair with larger interior angle will have greater effect on neighboring regions which is undesirable in mesh simplification. Therefore, the swapping of loops is excluded from consideration.

\subsection{Synthesis}

The whole process is summarized in Figure 8. As the data is loaded, where the height threshold $\delta$, interior angle $\theta$ and height parameter $\lambda$ are set to $180^{\circ}$ and 0 , respectively. The outer loop increases $\lambda$ from zero to $\delta$, while the inner loop decreases $\theta$ from $180^{\circ}$ to $\theta_{\min }$, which is chosen to be $150^{\circ}$ in the experiments. After the inner loop has completed searching and removing vertices by decreasing $\theta$, it is reset to $180^{\circ}$ and $\lambda$ is increased by adding previously defined $\Delta \lambda=\delta / k$ to $\lambda$. We employ this method to limit the iteration of outer loop to $k$ times. Sparse interval for $\lambda$ will prevent the inner loop from idly iterating when larger $\delta$ is used. The iteration number $k$ is set to 100 for the experiments. 


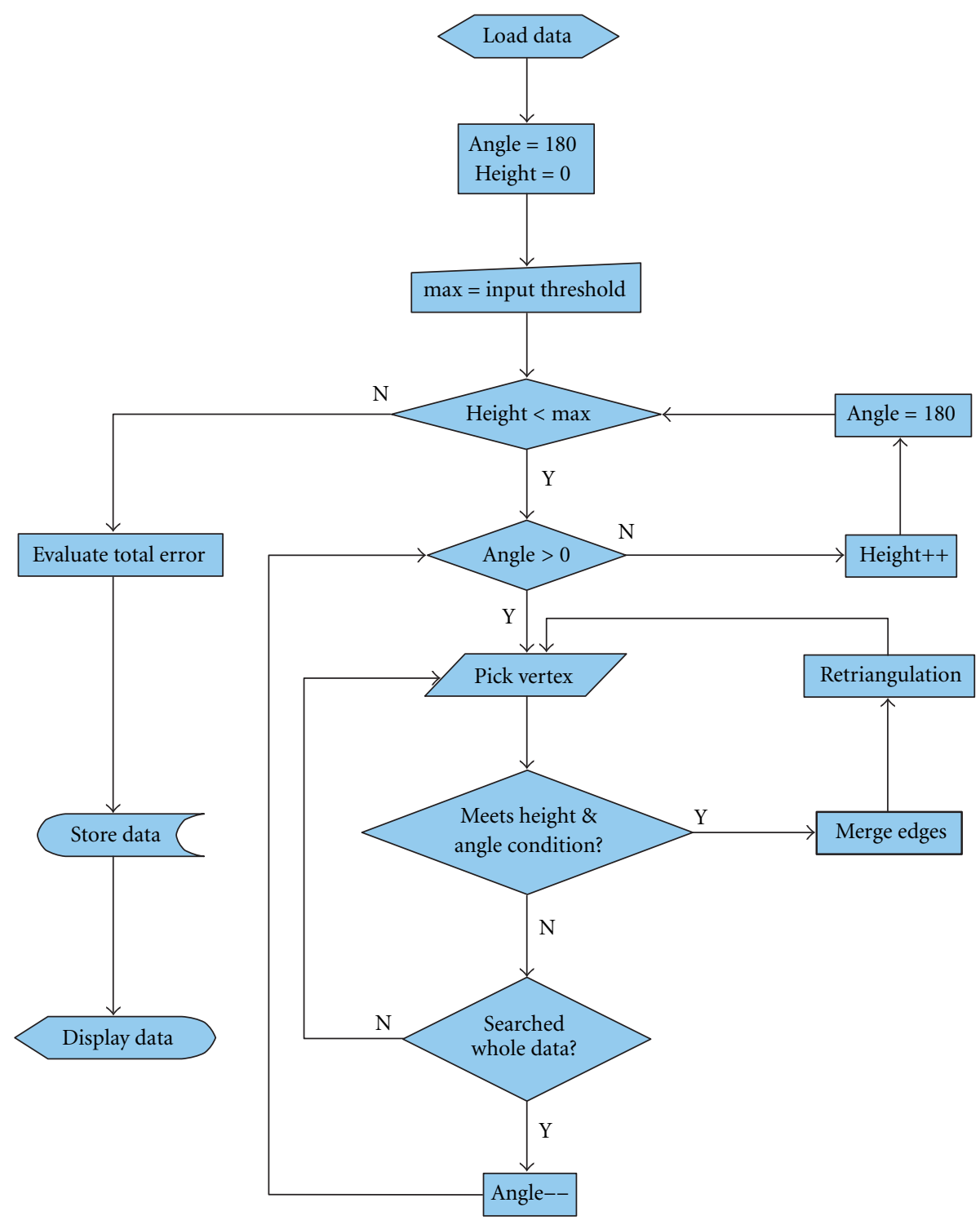

FIGURE 8: Block diagram for the proposed algorithm.

\subsection{Computation of error}

An error of decimated vertex is defined as its minimum distance to the newly created surface. Since the proposed algorithm does not move vertices' position, we only need to evaluate the errors caused by removing the vertices. Two error metrics, that is, $L^{\infty}$ and $L^{2}$ are widely used to evaluate the performance. The $L^{2}$ error norm is the root mean square (RMS) error, while the $L^{\infty}$ norm yields the maximum error bound. Both errors, defined by (1), are obtained for further analysis, where $N$ and $n$ are the original and removed numbers of vertices, respectively. Usually, the percentage of error to the diagonal length of original model's bounding box is used as a metric for comparison.

$$
\epsilon_{L^{2}}=\frac{\sqrt{\epsilon_{1}^{2}+\epsilon_{2}^{2}+\cdots+\epsilon_{n}^{2}}}{N}, \quad \epsilon_{L^{\infty}}=\max _{k=1}^{n} \epsilon_{k} .
$$

\subsection{Time complexity of the proposed algorithm}

Computational complexity mainly depends on the size of the data, because one of the bottle neck of the algorithm is the memory copying operations. When a vertex to be removed is found, all associated edges and triangles should be removed from its linked list. We simply generate a small temporary list for each, by copying the remaining edges and triangles, then swap the temporary list with the original one. Almost equal time is required when the hole is retriangulated to add edges and triangles to the linked list. The computational complexity could be alleviated, if this deletion process is optimized by dynamically allocating (deleting) spaces for the data. Therefore, the computational complexity is mainly dependent on (a) searching and (b) retriangulation process.

We search for the vertex list until appropriate vertex satisfying both height and angle condition is found. Statistically, 
when a linked-list of length $k$ has only one data that suites one's need, the average searching time, $s_{k}$ to find the suitable vertex is proportional to $(k+1) / 2$, given by

$$
s_{k} \propto \frac{1+2+\cdots+k}{k}=\frac{k+1}{2} .
$$

Retriangulation process requires for deletion and addition of triangles and edges. In average, when a vertex with six edges are deleted, which is the most popular case as shown in Figure 3, the required operations are (a) deletion of six triangles and six edges, and (b) addition of four new triangles and three new edges. Since removing or adding a triangle from a list of length $l$ requires $2(l-1)$ address copying operations, and the number of triangles and edges are proportional to the number of vertices by Euler's formula, the time required for deletion and addition of triangle is proportional to the vertex list of length $k$, given by

$$
r_{k} \propto \sum_{i=1}^{6} 4(k-i)+\sum_{i=3}^{6} 4(k-i)=40 k-156 .
$$

The first term is time for deletion of edges and triangles, respectively, while the second term is time for addition. The total time required for removing $n$ vertices from an original model with $N$ vertices is given by

$$
\begin{aligned}
T_{N}^{n} & =\sum_{k=N}^{N-n+1}\left(c_{1} s_{k}+c_{2} r_{k}\right) \\
& =c_{1} \frac{n(2 N-n+3)}{4}+c_{2} n(40 N-20 n-136),
\end{aligned}
$$

where $c_{1}$ and $c_{2}$ are scaling constants. Note that the constants would depend on the computing performance and programming efficiency. To show the validity of the time complexity analysis, we compare the experimental and theoretical computation time for the Face model in Figure 9. The scaling constants are found to be $c_{1}=-0.05302$ and $c_{2}=0.00069$, respectively, by the least mean square curve fitting method.

\section{EXPERIMENTS AND DISCUSSIONS}

We implement the proposed algorithm on Windows NT Server 4.0 with Pentium II $400 \mathrm{MHz}$ processor and $192 \mathrm{MB}$ memory. The results are flat shaded, in order to exaggerate the distinction of triangles. The Fan model in Figure 10 is a typical model produced by a CAD tool. The vertices and edges are almost optimally placed, which is almost impossible by actual modeling via range scanners. The original wireframe model is back-face culled in order to emphasize the placements of primitives. The simplification process faithfully preserves the unique characteristics of Fan's hole and wings. The result consists of only $19 \%$ of the original data, with tolerable errors provided in Table 1.

Figure 11 shows the hierarchy of Cow models from high to low resolution. The histogram indicates that vertices with high interior angles are mostly removed during the iteration. High curvature regions such as horn, ear, and nipples are well

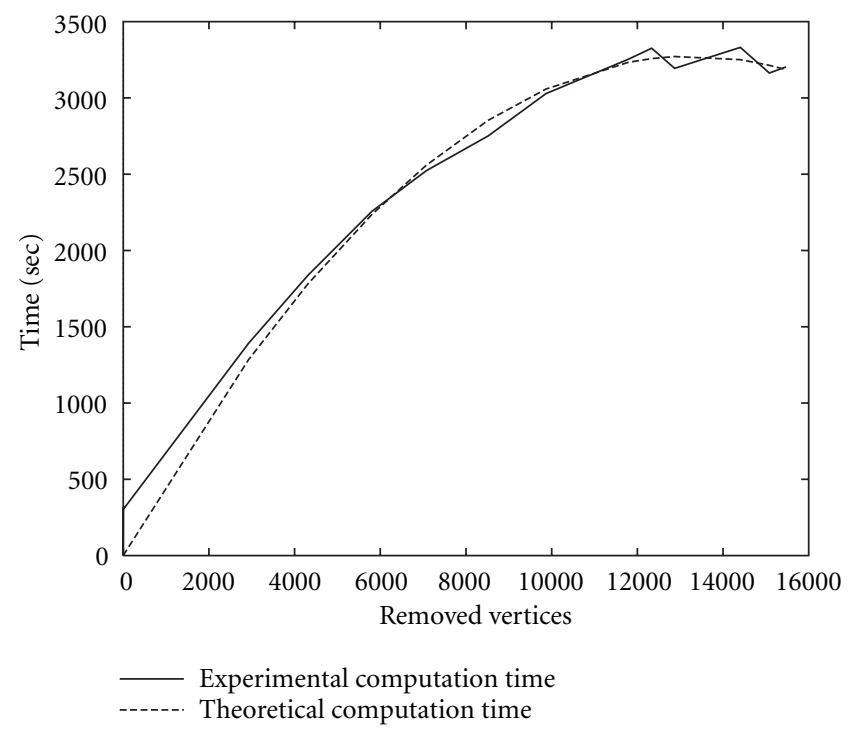

FIgURE 9: Time consumed for Face model of 16374 vertices.

TABLE 1: Summary of results on Fan model.

\begin{tabular}{lcccc}
\hline$\delta$ & No. of triangles & $L^{2}$ error $(\%)$ & $L^{\infty}$ error (\%) & Data ratio (\%) \\
\hline 0 & 2750 & 0 & 0 & 100 \\
2.0 & 1978 & 0.013 & 2.082 & 71.93 \\
2.3 & 1354 & 0.023 & 3.237 & 49.24 \\
3.0 & 1108 & 0.027 & 2.636 & 40.29 \\
5.0 & 530 & 0.043 & 6.369 & 19.27 \\
\hline
\end{tabular}

TABLE 2: Summary of results on Cow model.

\begin{tabular}{lcccc}
\hline$\delta$ & No. of triangles & $L^{2}$ error $(\%)$ & $L^{\infty}$ error (\%) & Data ratio (\%) \\
\hline 0 & 5804 & 0 & 0 & 100 \\
0.5 & 5652 & 0.001 & 0.745 & 97.38 \\
0.8 & 5096 & 0.003 & 1.047 & 87.80 \\
1.0 & 4620 & 0.004 & 1.320 & 79.60 \\
1.2 & 4216 & 0.005 & 1.320 & 72.64 \\
1.5 & 3454 & 0.007 & 1.523 & 59.51 \\
2.0 & 2668 & 0.009 & 1.820 & 45.97 \\
2.5 & 1978 & 0.013 & 2.676 & 34.08 \\
3.0 & 1538 & 0.015 & 2.526 & 26.50 \\
4.0 & 1136 & 0.019 & 3.480 & 19.57 \\
5.0 & 842 & 0.024 & 4.070 & 14.51 \\
\hline
\end{tabular}

preserved, while maintaining good equi-angularity as well. The results are summarized in Table 2.

Unlike the previous models, the Face model was acquired by laser range scanner, yielding a large amount of data. Due to its dense characteristics, most adjacent triangles are coplanar as can be seen from the initial histogram in Figure 12. 

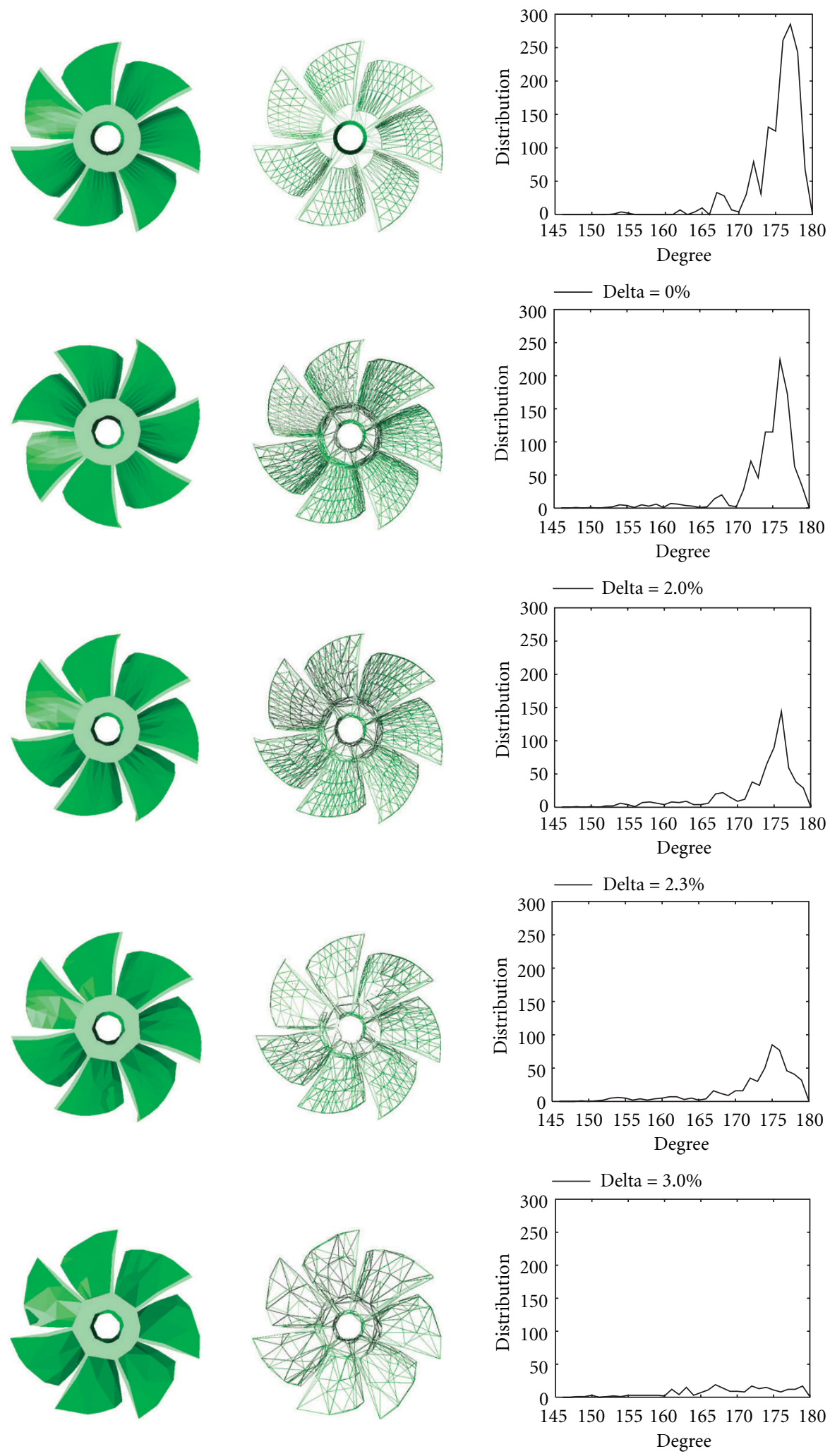

Delta $=5.0 \%$

Figure 10: Hierarchy of Fan models. 

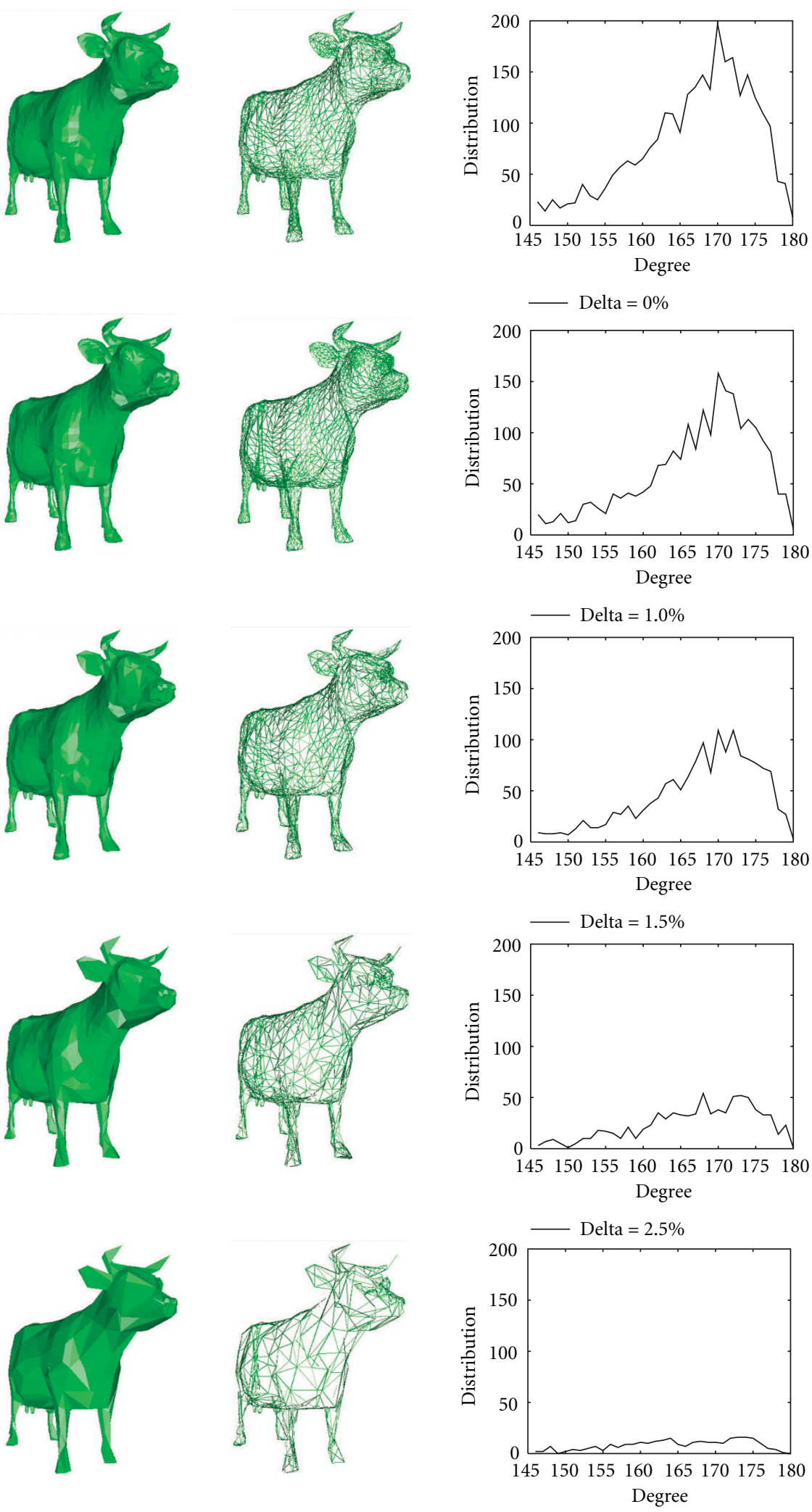

Delta $=5.0 \%$

FIgURE 11: Hierarchy of Cow models. 

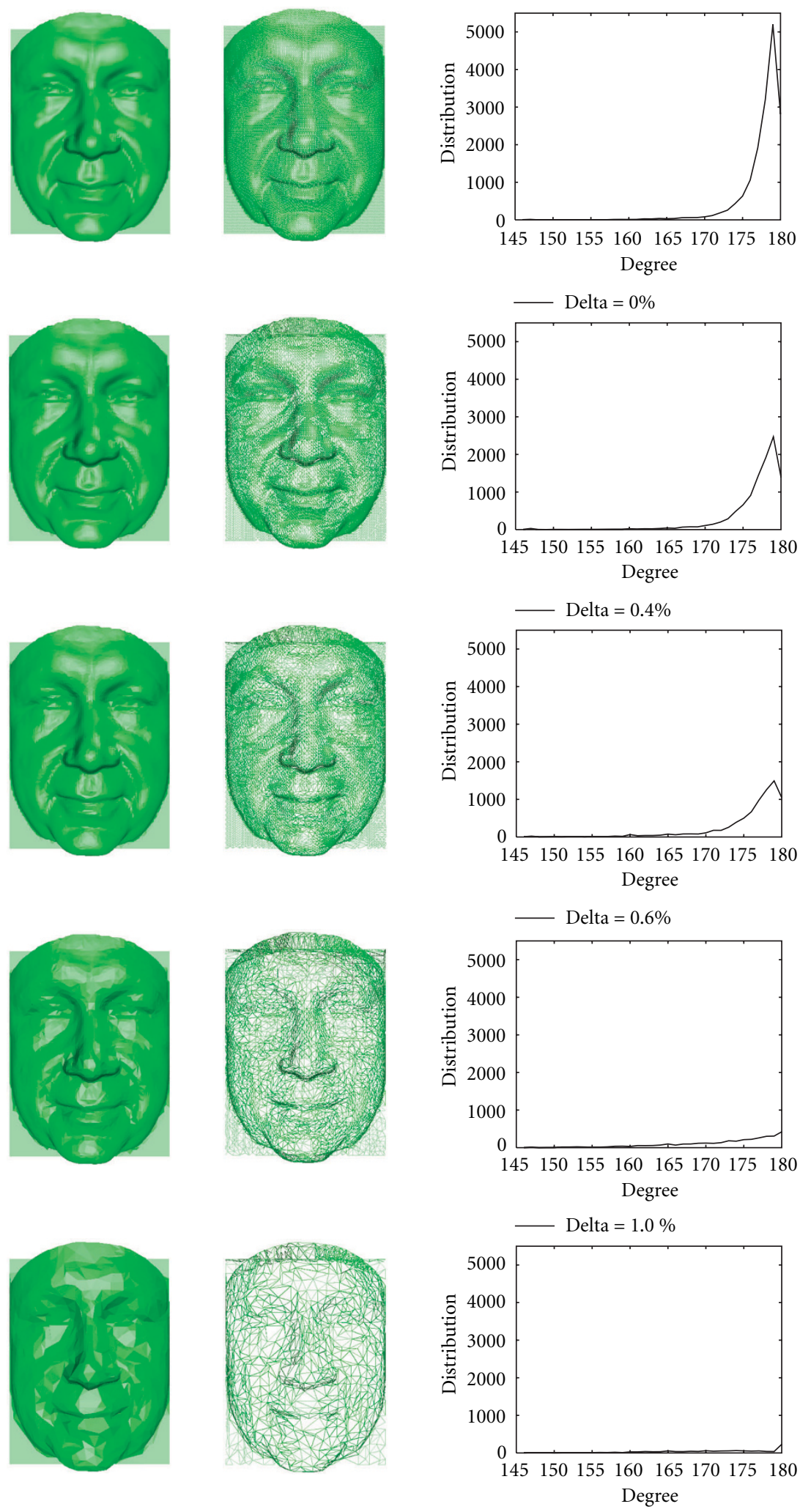

D Delta $=2.0 \%$

FIgURE 12: Hierarchy of Face models. 
TABLE 3: Summary of results on Face model.

\begin{tabular}{|c|c|c|c|c|}
\hline$\delta$ & No. of Triangles & $L^{2}$ error $(\%)$ & $L^{\infty}$ error $(\%)$ & Data ratio (\%) \\
\hline 0 & 32744 & 0 & 0 & 100 \\
\hline 0.36 & 26890 & 0.001 & 0.747 & 82.122 \\
\hline 0.37 & 24126 & 0.001 & 0.797 & 73.681 \\
\hline 0.4 & 21142 & 0.001 & 0.822 & 64.568 \\
\hline 0.5 & 18590 & 0.001 & 1.065 & 56.774 \\
\hline 0.6 & 15708 & 0.002 & 1.356 & 47.972 \\
\hline 1.0 & 7002 & 0.003 & 1.361 & 21.384 \\
\hline 1.5 & 3936 & 0.004 & 1.674 & 12.021 \\
\hline 2.5 & 1800 & 0.007 & 2.349 & 5.497 \\
\hline
\end{tabular}

TABLE 4: Summary of results on Knot model.

\begin{tabular}{lcccc}
\hline$\delta$ & No. of Triangles & $L^{2}$ error (\%) & $L^{\infty}$ error (\%) & Data ratio (\%) \\
\hline 0 & 76412 & 0.000 & 0.000 & 100 \\
0.1 & 73572 & 0.000 & 0.259 & 96.283 \\
0.2 & 64432 & 0.000 & 0.400 & 84.322 \\
0.3 & 45420 & 0.000 & 0.582 & 59.441 \\
0.35 & 35142 & 0.000 & 0.853 & 45.990 \\
0.4 & 24864 & 0.001 & 0.882 & 32.539 \\
0.45 & 17586 & 0.001 & 0.935 & 23.015 \\
0.7 & 10038 & 0.001 & 0.934 & 13.137 \\
0.8 & 7434 & 0.001 & 1.032 & 9.729 \\
\hline
\end{tabular}

Hence, a significant number of reduction takes place, even with small $\delta$ values. The low detailed parts of the surface are saved throughout the whole process.

The final model produced by interpolating a CAD model with high sampling rates contains 76412 triangles initially. A vertex with larger interior angle has been primarily removed, keeping vertices with small interior angles to preserve the global topology. The maximum error for final model is only $1.0 \%$ as shown in Figure 13 and Table 4, respectively.

To examine quantitative performance, we perform an experiment for Heckbert and Garland's algorithm [2]. The results are presented in Table 6 . We can find that the error performance is similar to or less than that of Tables 1,2, and 3 .

Optimal mesh simplification can be performed by fully sorting the order of removal after each iteration. Similar results may also be obtained by choosing very small value for $\Delta \lambda$. The interval of $\lambda$ significantly influences the output. If we set the $\Delta \lambda$ to greater magnitude, it is expected that the final result does not yield on uniform distribution in edge lengths, because the deterministic property of decimation could not be preserved. As a result, many less qualified vertices may be removed, prior to the more qualified ones.

However, the experiments for larger $\Delta \lambda$ yields interesting
TABLE 5: Summary of results on Cow model with $\Delta \lambda=\delta / 2$.

\begin{tabular}{lcccc}
\hline$\delta$ & No. of Triangles & $L^{2}$ error $(\%)$ & $L^{\infty}$ error (\%) & Data ratio (\%) \\
\hline 0 & 5804 & 0 & 0 & 100 \\
0.5 & 5650 & 0.001 & 0.727 & 97.35 \\
1.0 & 4598 & 0.004 & 1.320 & 79.22 \\
1.5 & 3532 & 0.007 & 1.479 & 60.85 \\
2.0 & 2862 & 0.009 & 1.890 & 49.31 \\
2.5 & 2214 & 0.011 & 2.177 & 38.15 \\
3.0 & 1846 & 0.013 & 2.960 & 31.81 \\
3.5 & 1496 & 0.016 & 3.125 & 25.78 \\
4.0 & 1406 & 0.017 & 3.739 & 24.22 \\
5.0 & 1164 & 0.020 & 3.876 & 20.06 \\
\hline
\end{tabular}

TABLE 6: Comparison of Heckbert and Garland algorithm.

\begin{tabular}{|c|c|c|c|c|}
\hline Model & No. of Triangles & $L^{2}$ error $(\%)$ & $L^{\infty}$ error $(\%)$ & Data ratio $(\%)$ \\
\hline Fan & 1978 & 0.077 & 2.791 & 71.93 \\
\hline Fan & 1354 & 0.101 & 3.214 & 49.24 \\
\hline Fan & 1108 & 0.112 & 3.657 & 40.29 \\
\hline Fan & 530 & 0.211 & 6.477 & 19.27 \\
\hline Cow & 4620 & 0.718 & 8.12 & 79.60 \\
\hline Cow & 3454 & 0.755 & 8.19 & 59.51 \\
\hline Cow & 1978 & 0.733 & 8.00 & 34.08 \\
\hline Cow & 842 & 0.795 & 8.11 & 14.51 \\
\hline Face & 7002 & 0.042 & 7.42 & 21.38 \\
\hline Face & 1800 & 0.058 & 7.28 & 5.497 \\
\hline
\end{tabular}

results. By choosing large value for $\Delta \lambda$, the total number of iterations of inner loop decreases, since the outer loop iterates according to the size of $\Delta \lambda$. Therefore, total execution time greatly reduces, since the number of iterations is also one of the bottle neck of the proposed algorithm. It is argued that larger $\Delta \lambda$ has little influence on the quantitative properties, resulting in nonuniform distribution in edge lengths. To show the effect of larger $\Delta \lambda$, we present the results obtained by setting $\Delta \lambda=\delta / 2$ in Table 5 . By comparing the seventh row of Table 2 and the fifth row of Table 5, we can find that two results show the comparable errors with almost the same number of triangles. However, the execution time is reduced to $40 \%$, due to fewer iterations. This is because the initial triangular mesh does not contain vertices with long edges. However, the visual deterioration is observed as the total number of face reduced to around 1000 triangles, in which the edge lengths' distribution is nonuniform. Figure $14 \mathrm{com}-$ pares both results obtained by different $\Delta \lambda$.

Finally, it is interesting to note a related work in discrete contour evolution [13], which are developed for 2D shape 

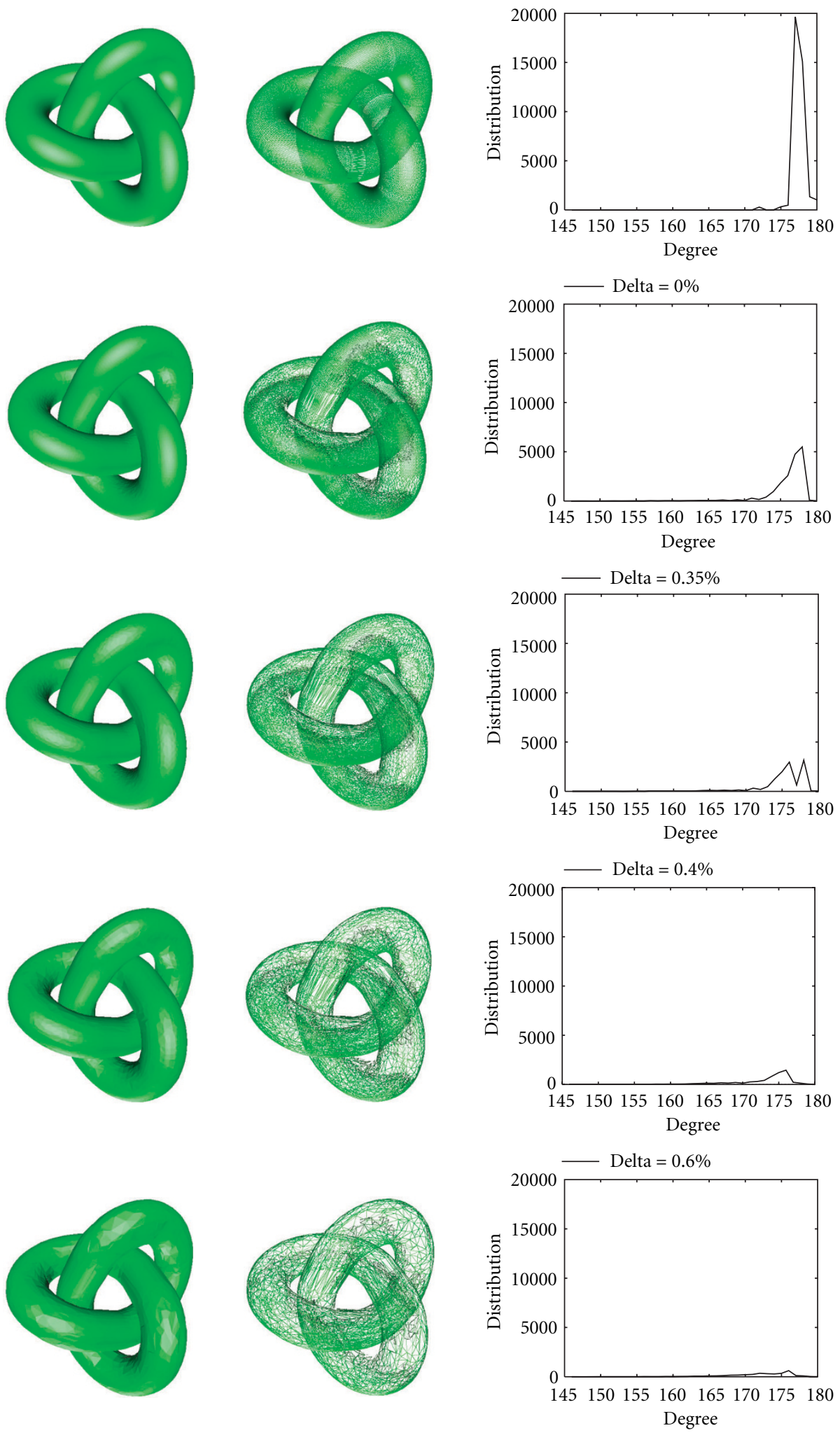

- Delta $=0.8 \%$

FIGURE 13: The simplification results of Knot model. 


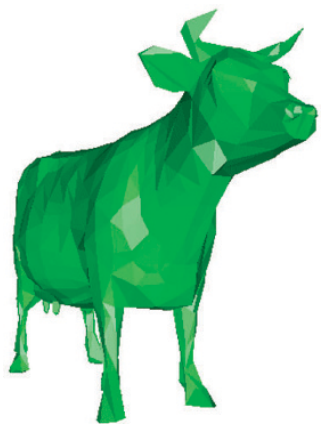

(a) $\Delta \lambda=\delta / 100$.

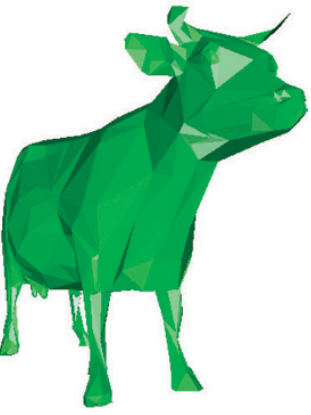

(b) $\Delta \lambda=\delta / 2$.
FIGURE 14: Visual comparison of results.

decomposition. They proposed a relevance measure based on an interior angle and edge length. We believe that our scheme is very similar to [13], although each of these has different aims. Thus, our further research topics include multiresolution modeling using the relevance measure.

\section{CONCLUSION}

We have presented an edge-based vertex removal algorithm to simplify triangulated objects. The removal of a vertex is determined by the edges, connected to other vertices. If the interior angle of edge pair is larger than the pre-specified threshold, the edge pair will be considered as a semi-linear line, and the vertex is removed. An additional height constraint was introduced to prevent the decimation of long edge pairs, which yield relatively large error and degrade the topology. This criterion makes the proposed algorithm much faster than the conventional algorithms, which remove a vertex by estimating the post error caused by removing.

The execution interval of the proposed algorithm decreases linearly as the vertices are removed, while that of the conventional approaches increase exponentially. The performance was comparable, in spite of using such a simple measure for simplification. The computational complexity relied heavily on the number of iterations of two nested loops. However, the computational complexity could be alleviated by reducing the interval of height parameter $(\Delta \lambda)$ and angle parameter $(\Delta \theta)$. Observations showed that the quantitative performance is not significantly affected by the choice $\Delta \lambda$.

However, further research should be necessary on the optimal selection for $\Delta \lambda$. This value could be adaptively updated according to the angle histogram of original data, or the height parameter itself should be increased from linear to exponentially decreasing manner. This is of special interest for simplification of highly detailed meshes, since large $\Delta \lambda$ does not affect the output of the earlier stages. The minimum angle parameter $\theta_{\min }$ and the interval $\Delta \theta$ also should be carefully selected for better results.

\section{ACKNOWLEDGMENT}

This work was supported by the Korea Research Foundation Grant. (KRF-2001-041-E00258).

\section{REFERENCES}

[1] J. Li and J. Kuo, "A dual graph approach to 3D triangular mesh compression," in Proc. IEEE International Conference on Image Processing, pp. 891-894, Chicago, Ill, USA, October 1998.

[2] P. Heckbert and M. Garland, "Survey of surface approximation algorithms," Tech. Rep. CMU-CS-95-194, Carnegie Mellon University, 1995.

[3] T. Gieng, B. Hamann, K. Joy, G. Schussman, and I. Trotts, "Constructing hierarchies for triangle meshes," IEEE Transactions on Visualization and Computer Graphics, vol. 4, no. 2, pp. 145-161, 1998.

[4] J. Cohen, A. Varshney, D. Minocha, et al., "Simplification envelopes," in Computer Graphics (Proc. SIGGRAPH '96), pp. 119-128, New Orleans, La, USA, August 1996.

[5] W. Lorensen, W. Schroeder, and J. Zarge, "Decimation of triangular meshes," in Computer Graphics (Proc. SIGGRAPH '92), pp. 65-70, Chicago, Ill, USA, July 1992.

[6] M. Soucy and D. Laurendeau, "Hierarchical surface triangulation of range data," in Canadian Conference on Electrical and Computer Engineering, pp. 4.4.1-4.4.4, Québec, QC, Canada, September 1991.

[7] M. Soucy and D. Laurendeau, "Multiresolution surface modeling based on hierarchical triangulation," Computer Vision and Image Understanding, vol. 63, no. 1, pp. 1-14, 1996.

[8] R. Sibson, "Locally equi-angular triangulation," Computer Journal, vol. 21, no. 3, pp. 243-245, 1978.

[9] A. Guéziec, "Surface simplification using quadric error metrics," in Computer Graphics (Proc. SIGGRAPH '97), pp. 209216, Los Angeles, Calif, USA, August 1997.

[10] H. Hoppe, T. DeRose, J. McDonald, and W. Stuetzle, "Mesh optimization," in Computer Graphics (Proc. SIGGRAPH '93), pp. 19-26, Anaheim, Calif, USA, August 1993.

[11] A. E. Johnson and M. Hebert, "Control of polygonal mesh resolution for 3-D computer vision," Graphical Models and Image Processing, vol. 60, no. 4, pp. 261-285, 1998.

[12] T. Fang and L. Piegl, "Delaunay triangulation in three dimensions," IEEE Computer Graphics and Applications, vol. 15, no. 5, pp. 62-69, 1995.

[13] L. J. Latecki and R. Lakämper, "Convexity rule for shape decomposition based on discrete contour evolution," Computer Vision and Image Understanding, vol. 73, no. 3, pp. 441-454, 1999.

Il Dong Yun received his B.S., M.S., and Ph.D. degrees in electrical engineering from Seoul National University, Seoul, Korea, in 1989, 1991, and 1996, respectively. From 1996 to 1997, he was employed at the Daewoo Electronics Inc., Seoul, Korea. In 1997, he joined the School of Electronics and Information Engineering at Hankuk University of Foreign Studies, as a faculty member, where he is currently an Assistant Professor. His research interests include object recognition, texture segmentation, and 3D shape reconstruction, segmentation, and description. 
Kyowoong Choo received his B.S. and M.S. degrees in electrical engineering from Seoul National University in 1997 and 1999, respectively. In 1999, he joined the DTV Laboratory of LG Electronics as a Research Engineer and worked on Digital Broadcasting Receivers. In 2002, he joined Motorola Korea, where he is currently a Senior Research Engineer in the Personal Communication Sector. His current research fields are VOD and video phone services on wireless networks.

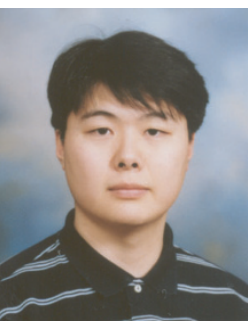

Sang Uk Lee received his B.S. degree from Seoul National University, Seoul, Korea, in 1973, the M.S. degree from Iowa State University, Ames in 1976, and Ph.D. degree from the University of Southern California, Los Angeles, in 1980, all in electrical engineering. From 1980 to 1981 , he was with the General Electric Company, Lynchburg, Va, working on the development of digital mobile radio. From 1981 to 1983 , he was a

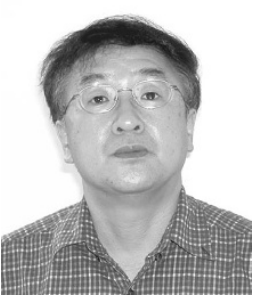
Member of Technical Staff, M/A-COM Research Center, Rockville, Md. In 1983, he joined the Department of Control and Instrumentation Engineering at Seoul National University as an Assistant Professor, where he is now a Professor at the School of Electrical Engineering and Computer Science. Currently, he is also affiliated with the Automation and Systems Research Institute and the Institute of New Media and Communications at Seoul National University. His current research interests are in the areas of image and video signal processing, digital communication, and computer vision. He served as an Editor-in-Chief for the Transaction of the Korean Institute of Communication Science from 1994 to 1996. Dr. Lee is currently a Member of the Editorial Board of the Journal of Visual Communication and Image Representation and an Associate Editor for IEEE Transactions on Circuits and Systems for Video Technology. He is a member of Phi Kappa Phi. 